Pacific Journal of Mathematic 


\section{ASYMPTOTIC RELATIONS BETWEEN SYSTEMS OF DIFFERENTIAL EQUATIONS}

CHOY-TAK TAAM

1. Introduction. A. Wintner [1], N. Levinson [2], H. Weyl [3] and others have obtained interesting asymptotic relations between the solutions of a given system of differential equations and those of an approximate system. In their investigations the solutions of the approximate system of differential equations are assumed to be bounded. In this paper we consider the asymptotic problems of the solutions from a more general point of view, given only certain order relations satisfied by the solutions of the approximate system. The method we shall use is to study an associated system of integral equations which yields the asymptotic relations between the solutions of the perturbed and unperturbed equations. With the aid of the Phragmén-Lindelöf Theorems [4], our results can be easily extended to the complex domain.

2. Asymptotic relations in the real domain. Consider the system of differential equations written in the vector form

$$
\frac{d y}{d x}=A(x) y+f(x, y),
$$

where $A(x)=\left\|a_{i j}(x)\right\|$ is a $n \times n$ matrix and $y$ and $f(x, y)$ are respectively column vectors with components $y^{(i)}$ and $f^{(i)}(x, y), i=1,2, \cdots, n$. Defining the norms $\|y\|,\|A\|$ of vectors $y$ and matrices $A$ by

$$
\|y\|=\sum_{i=1}^{n}\left|y^{(i)}\right|,\|A\|=\sum_{i, j=1}^{n}\left|a_{i j}\right|,
$$

it is easy to verify that

$$
\begin{array}{ll}
\left\|y_{1}+y_{2}\right\| \leqq\left\|y_{1}\right\|+\left\|y_{2}\right\|, & \left\|A_{1}+A_{2}\right\| \leqq\left\|A_{1}\right\|+\left\|A_{2}\right\|, \\
\left\|A_{1} A_{2}\right\| \leqq\left\|A_{1}\right\|\left\|A_{2}\right\|, & \|A y\| \leqq\|A\|\|y\| .
\end{array}
$$

In this section we assume that $a_{i j}(x)$ and $f^{(i)}(x, y)$ (for each fixed complex $y$ ) are complex-valued functions of the real variable $x$ belonging to $L(0, R)$ for every positive $R$. Furthermore we assume that for each $x \geqq 0, f^{(i)}(x, y)$ is a continuous function of $y$ for all complex $y$ and $f(x, y)$ satisfies the Lipschitz condition

Received August 20, 1953. Presented to the American Mathematical Society, December $28,1953$. 


$$
\left\|f\left(x, y_{1}\right)-f\left(x, y_{2}\right)\right\| \leqq g(x)\left\|y_{1}-y_{2}\right\|
$$

for all complex $y_{1}$ and $y_{2}, g(x)$ being of the class $L(0, R)$ for every positive $R$. (If all the functions $a_{i j}(x)$ and $f^{(i)}(x, y)$ are real and only real-valued solutions are considered, it is enough to assume that $f^{(i)}(x, y)$ is continuous for all real $y$ and (2.2) holds for all real $y_{1}$ and $y_{2}$.)

By a solution of (2.1) we mean a vector function $y(x)$ which is absolutely continuous and satisfies the equation (2.1) almost everywhere. For the existence and uniqueness of solutions, see [5, Sections 68.3 and 68.5].

In this section we shall establish two asymptotic relations between the solutions of (2.1) and those of the approximate equation

$$
d y=A(x) y \text {. }
$$

Equation (2.3) has $n$ linearly independent vector solutions $y_{i}, i=1$, $2, \cdots, n$. Let $Y$ be the $n \times n$ matrix whose columns are the $n$ vector solutions $y_{i}, y_{i}$ being so chosen to satisfy the initial condition $Y(0)=I$. $Y$ is non-singular and has an inverse $Y^{-1}$. Since each column of $Y$ is a solution of (2.3) it is clear that $Y$ satisfies

$$
d y=A(x) Y, Y(0)=I
$$

If $\operatorname{tr} A$ denotes the sum of the diagonal elements of $A$, it is well-known [6] that

$$
\operatorname{det} Y(x)=\exp \left(\int_{0}^{x} \operatorname{tr} A(t) d t\right)
$$

For convenience we first establish the following results.

LEMma 1. Let

(a) $y(x)$ be a solution of $(2.1), y(0)=c$,

(b) $Y(x)$ be the matrix solution of (2.4).

Then $y(x)$ satisfies the vector integral equation

$$
y(x)=Y(x) c+\int_{0}^{x} Y(x) Y^{-1}(t) f(t, y(t)) d t .
$$

Proof. Set $y(x)=Y(x) z(x)$. Then a substitution into (2.1) gives

$$
\frac{d Y}{d x} z+Y_{d x}^{d z}=A Y z+f
$$

Using (2.4), we have 


$$
d z=Y^{-1} f
$$

or

$$
z(x)=c+\int_{0}^{x} Y^{-1}(t) f(t, y(t)) d t .
$$

Multiplication of (2.7) by $Y(x)$ gives the result (2.5).

The following lemma is due to R. Bellman.

Lemma 2. Let $u(x)$ and $v(x)$ be real-valued functions defined for $x \geq a$, both being non-negative. If

(a) $v(x)$ and $u(x) v(x)$ belong to $L(a, R)$ for every $R \geqq a$.

(b) $u(x) \leqq M+\int_{a}^{x} u(t) v(t) d t, M>0,(x \geqq a)$, then

$$
u(x) \leqq M e^{\int_{a}^{x} a^{(t) d t}},
$$

Proof. Multiplication of (b) by $v(x)$ gives

$$
u(x) v(x) \leqq v(x)\left(M+\int_{a}^{x} u(t) v(t) d t\right)
$$

Divide both sides of (2.8) by $M+\int_{a}^{x} u(t) v(t) d t$ and integrate the result from $a$ to $x$, obtaining

$$
\log \left(M+\int_{a}^{x} u(t) v(t) d t\right) \leqq \log M+\int_{a}^{x} v(t) d t .
$$

Then lemma follows from (b) and (2.9).

We first prove the following asymptotic relation. $ת a$ denotes the real part of a complex number $a$.

\section{Theorem 1. Let}

(a) $\|Y(x)\|=O(h(x))$ as $x \rightarrow \infty, h(x)$ being measurable on $0 \leqq x<\infty$,

(b) $\|f(x, 0)\|[h(x)]^{n-1} \exp \left[-\mathscr{R} \int_{0}^{x} \operatorname{tr} A(t) d t\right]$ and

$$
g(x)[h(x)]^{n} \exp \left[-\mathscr{R} \int_{1}^{x} \operatorname{tr} A(t) d t\right] \text { belong to } L(0, \infty) \text {. }
$$

Then, for each solution $y(x)$ of $(2.1),\|y(x)\|=O(h(x))$ as $x \rightarrow \infty$ and there is a solution $Y(x) c$ of (2.3) such that 
$(2.10)$

$$
y(x)=Y(x)(c+\varepsilon(x)),
$$

where $\|\varepsilon(x)\|$ tends to zero as $x \rightarrow \infty$.

Proof. According to Lemma 1,y(x) satisfies the integral equation

$$
y(x)=Y(x) c+\int_{0}^{x} Y(x) Y^{-1}(t) f(t, y(t)) d t .
$$

It follows that

$$
\|y(x)\| \leq\|Y(x)\|\|c\|+\int_{0}^{x}\|Y(x)\|\left\|Y^{-1}(t)\right\|\|f(t, y(t))\| d t .
$$

Since (2.2) implies that

$$
\|f(x, y)\| \leqq g(x)\|y\|+\|f(x, 0)\|
$$

and $\|Y(x)\|$ does not vanish, one obtains from (2.12)

$$
\underset{\|Y(x)\|}{\|y(x)\|} \leq\|c\|+\int_{0}^{x}\left\|Y^{-1}(t)\right\|(g(t)\|y(t)\|+\|f(t, 0)\|) d t .
$$

Since each component of $Y^{-1}(x)$ is the cofactor of the corresponding component in $\operatorname{det} Y(x)_{t}$ divided by $\operatorname{det} Y(x), Y(x)_{t}$ being the transpose of $Y(x)$, it is clear that as $x \rightarrow \infty$

$$
\left\|Y^{-1}(x)\right\|=O\left([h(x)]^{n-1} \exp \left[-\int_{0}^{x} \operatorname{tr} A(t) d t\right]\right) .
$$

From (b), it follows that as $x \rightarrow \infty$

$$
\int_{0}^{x}\left\|Y^{-1}(t)\right\|\|f(t, 0)\| d t=O(1) .
$$

From (2.14) we then have

$$
\|y(x)\| \leqq M+\int_{0}^{x} g(t)\left\|Y^{-1}(t)\right\|\|Y(t)\| \|_{\|Y(t)\|}^{\|y(t)\|} d t
$$

for some constant $M$. An appeal to Lemma 2 yields

$$
\|y(x)\| \leq M \exp \left(\int_{0}^{x} g(t)\left\|Y^{-1}(t)\right\|\|Y(t)\| d t\right) .
$$

Conditions (a), (b) and (2.15) ensure that the integral in (2.18) is bounded for $x \geq 0$ and consequently as $x \rightarrow \infty$

$$
\|y(x)\|=O(h(x)) .
$$

Since 


$$
\int_{0}^{x}\left\|Y^{-1}(t)\right\|\|f(t, y(t))\| d t \leqq \int_{0}^{x}\left\|Y^{-1}(t)\right\|(g(t)\|y(t)\|+\|f(t, 0)\|) d t
$$

and the integral on the right hand side is convergent (by virtue of conditions (a), (b), (2.15) and (2.19)), the integral

$$
\int_{0}^{\infty} Y^{-1}(t) f(t, y(t)) d t
$$

exists. Splitting the integral in (2.11) into two, one integrating from 0 to $\infty$ and the other from $x$ to $\infty$, we have

$$
y(x)=Y(x)(c+\varepsilon(x))
$$

for an appropriate constant $c$, where

$$
\varepsilon(x)=-\int_{x}^{\infty} Y^{-1}(t) f(t, y(t)) d t
$$

This completes the proof of Theorem 1 .

Given stronger conditions, we can show that $\|Y(x) \varepsilon(x)\|$ tends to zero as $x$ tends to infinity.

THEOREM 2. Let

(a) $\|Y(x)\|=O(h(x))$ as $x \rightarrow \infty, h(x)$ being non-decreasing on $0 \leqq x<\infty$,

(b) $\|f(x, 0)\|[h(x)]^{n} \exp \left[-\mathscr{R} \int_{0}^{x} \operatorname{tr} A(t) d t\right]$ and

$$
g(x)[h(x)]^{n+1} \exp \left[-\mathscr{R} \int_{0}^{x} \operatorname{tr} A(t) d t\right] \text { belong to } L(0, \infty) \text {. }
$$

Then for each solution $y(x)$ of (2.1) there is a solution $Y(x) c$ of (2.3) such that

$$
y(x)=Y(x) c+\varepsilon(x),
$$

where $\|\varepsilon(x)\|$ tends to zero as $x$ tends to infinity.

Proof. Since the conditions of Theorem 1 are satisfied, we obtain at once the relation (2.22) with

$$
\varepsilon(x)=-Y(x) \int_{x}^{\infty} Y^{-1}(t) f(t, y(t)) d t .
$$

Clearly

$$
\begin{aligned}
\|\varepsilon(x)\| & \leqq \int_{x}^{\infty}\|Y(x)\|\left\|Y^{-1}(t)\right\|\|f(t, y(t))\| d t \\
& \leq M \int_{x}^{\infty} h(t)\left\|Y^{-1}(t)\right\|(g(t)\|y(t)\|+\|f(t, 0)\|) d t
\end{aligned}
$$


for some constant $M$, the existence of the last integral being guaranteed by the conditions (b). It follows that $\|s(x)\|$ tends to zero as $x$ tends to infinity. This completes the proof.

Remark. Under the conditions of Theorem 2, the singular integral equation

$$
y(x)=Y(x) c-Y(x) \int_{x}^{\infty} Y^{-1}(t) f(t, y(t)) d t
$$

defines actually a one-to-one correspondence between the solutions of (2.1) and (2.3) such that the corresponding solutions are asymptotically equivalent in the sense of Theorem 2. The previous statement remains true if "Theorem 2 " is replaced by "Theorem 1 " provided $h(x)$ is bounded on $0 \leqq x \leqq x_{0}$ for every positive $x_{0}$. To see this it is sufficient to prove the existence and uniqueness of the solution of the integral equation (2.23) for an arbitrary but fixed $c$, the result then follows from the fact that a solution of (2.23) is a solution of (2.1).

Define $y_{0}(x)=0$ and

$$
y_{n}(x)=Y(x) c-Y(x) \int_{x}^{\infty} Y^{-1}(t) f\left(t, y_{n-1}(t)\right) d t, \quad n=1,2,3, \cdots .
$$

If $\left\|y_{n-1}(x)\right\|=O(h(x))$, the integral in (2.24) exists. It follows that $\left\|y_{n}(x)\right\|=O(h(x))$ and the integral in $(2.24)$ exists for all $y_{n-1}(x)$. If

$$
H(x)=\int_{x}^{\infty}[h(t)]^{n} g(t) \exp \left[-\mathscr{R} \int_{0}^{t} \operatorname{tr} A(u) d u\right] d t,
$$

it is easy to verify that

$$
\left\|y_{n+1}(x)-y_{n}(x)\right\| \leqq M^{\because n+1} h(x) \begin{gathered}
{[H(x)]^{n}} \\
n !
\end{gathered} \quad(n=0,1,2, \cdots)
$$

for an appropriate constant $M$. It follows that $y_{n}(x)$ converges to a limit $y(x)$ uniformly on $0 \leqq x \leqq x_{0}$ for every positive $x_{0}$. Clearly $\|y(x)\|=$ $O(h(x)),\left\|y_{n}(x)\right\|=O(h(x))$ uniformly in $n$ and $y(x)$ satisfies (2.23). Suppose that (2.23) has another solution $z(x)$, then $\|z(x)\|=O(h(x))$ and

$$
\|y(x)-z(x)\| \leqq\|Y(x)\| \int_{x}^{\infty}\left\|Y^{-1}(t)\right\| g(t)\|y(t)-z(t)\| d t
$$

or

$$
\|y(x)-z(x)\| \leqq M^{3} h(x) H(x)
$$

for some constant $M$. Substituting (2.28) into (2.27) and repeating the process, we have 


$$
\|y(x)-z(x)\| \leqslant M^{2 n+1} h(x) \stackrel{[H(x)]^{n}}{n !}
$$

for every $n$. (2.29) implies that $y(x)$ and $z(x)$ are equal and hence the solution of (2.23) is unique.

3. Asymptotic relations in the complex domain. In this section the results of $\S 2$ are extended to the complex domain for a system of linear differential equations. In $\S 2$ the success of the method depends on the Lipschitz condition (2.2). But if $f(x, y)$ is an analytic function of the complex variables $x$ and $y$ for $x$ in a region $R$ and all $y$ and satisfies the Lipschitz condition

$$
\left\|f\left(x, y_{1}\right)-f\left(x, y_{2}\right)\right\| \leqq g(x)\left\|y_{1}-y_{2}\right\|
$$

for all $y_{1}$ and $y_{2}$, it is necessary that $f(x, y)$ is a polynomial of degree 1 in $y$ or containing no $y$. If $f(x, y)$ is not analytic for all $y$, it is not clear to the author how this problem may be attacked in the complex domain.

For convenience we use $z$ as the independent variable and write $z=x+i y=r \exp (i \theta)$, where $x, y, r$ and $\theta$ and real and $r$ is non-negative.

We need the following Phragmén-Lindelöf theorems.

Let $R_{1}$ be the strip of the complex plane

$$
x \geq 0, \quad y_{1} \leqq y \leqq y_{2}, \quad y_{2}-y_{1}=a^{-1} \pi,
$$

and $R_{2}$ the sector

$$
r \geqq 0, \quad \theta_{1} \leqq \theta \leqq \theta_{2}, \quad \theta_{2}-\theta_{1}=a^{-1} \pi
$$

LEMma 3. Let $u(z)$ be an analytic function in $R_{1}$. If

(a) $|u(z)| \leqq M$ in $R_{1}$ for $x=0, y=y_{1}$ and $y=y_{2}$,

(b) $u(z)=O\left(e^{N e^{b . x}}\right)$ as $x \rightarrow \infty$, uniformly in $y_{1} \leqq y \leqq y_{2}$ for some constants $b$ and $N, b<a$,

then $|u(x)| \leqq M$ in $R_{1}$.

Lemma 4. Let $u(z)$ be analytic in $R_{1}$. If

(a) $u\left(x+i y_{k}\right)$ tends to a limit $a_{k}$ as $x \rightarrow \infty$, $k=1,2$,

(b) $u(z)$ is bounded in $R_{1}$, then as $z \rightarrow \infty u(z)$ tends to a limit a uniformly in $R_{1}$ and $a=a_{1}=a_{2}$.

LeMma 5. Let $u(z)$ be analytic in $R_{2}$. If

(a) $|u(z)| \leqq M$ in $R_{2}$ for $\theta=\theta_{1}$ and $\theta=\theta_{2}$,

(b) $u(z)=O\left(e^{N r^{b}}\right)$ as $r \rightarrow \infty$, uniformly in $\theta_{1} \leqq \theta \leqq \theta_{2}$ for some constants $b$ and $N, b<a$,

then $|u(z)| \leqq M$ in $R_{\text {. }}$. 
Lemma 6. Let $u(z)$ be analytic in $R_{2}$. If

(a) $u\left(r e^{i \theta_{k}}\right)$ tends to a limit $a_{k}$ as $r \rightarrow \infty$, $k=1,2$,

(b) $u(z)$ is bounded in $R_{2}$, then as $z \rightarrow \infty u(z)$ tends to a limit a uniformly in $R_{2}$ and $a=a_{1}=a_{2}$.

For proof of these theorems, see [4].

Consider the system of linear diffential equations written in the vector form

$$
\frac{d w}{d z}=(A(z)+B(z)) w+f(z)
$$

where $\left.A(z)=\| a_{i j}(z)\right)^{\prime}$ and $B(z)=\left\|b_{i j}(z)\right\|$ are $n \times n$ matrices and $w$ and $f(z)$ column vectors with components $w^{(i)}$ and $f^{(i)}(z)$ respectively, $i=1,2, \cdots n$. We assume that $a_{i j}(z), b_{i j}(z)$ and $f^{(i)}(z)$ are single-valued analytic functions in the regions with which we shall be concerned in this section.

Let $W(z)$ be a matrix solution of

$$
\frac{d W}{d z}=A(z) W, W\left(z_{0}\right)=I .
$$

We shall establish asymptotic relations between the solutions of (3.3) and those of the approximate system

$$
d w=A(z) w
$$

Write

$$
\begin{aligned}
& H(x, y)=H\left(x, y, x_{0}\right)=\int_{x_{0}}^{x}[h(x, y)]^{n}\|B(z)\| \exp \left[-\mathscr{L} \int_{z_{0}}^{z} \operatorname{tr} A(u) d u\right] d x, \\
& K(x, y)=K\left(x, y, x_{0}\right)=\int_{x_{j}}^{x}[h(x, y)]^{n-1}\|f(z)\| \exp \left[-\mathscr{C} \int_{z_{0}}^{z} \operatorname{tr} A(u) d u\right] d x,
\end{aligned}
$$

where $z=x+i y, z_{0}=x_{0}+i y_{0}, z_{0}$ being in $R_{1}$.

THEOREM 3. Let

(a) $\|W(z)\|=O(h(x, y))$ as $x \rightarrow \infty$ for $y=y_{1}$ and $y=y_{2}, \quad\|W(z)\|=$ $O\left(h(x, y) e^{b x}\right)$ as $x \rightarrow \infty$, uniformly in $y_{1} \leqq y \leqq y_{2}$, where $b<a$, $y_{2}-y_{1}=a^{-1} \pi, h(x, y)=|h(z)|$ and $h(z)$ is analytic in $R_{1}$,

(b) $H(x, y)=O(1)$ as $x \rightarrow \infty$ for $y=y_{1}$ and $y=y_{2}, H(x, y)=O\left(e^{b x}\right)$ as $x \rightarrow \infty$, uniformly in $y_{1} \leqq y \leqq y_{2}$,

(c) $K(x, y)=O(1)$ as $x \rightarrow \infty$ for $y=y_{1}$ and $y=y_{2}, K(x, y)=O\left(e^{e^{b x}}\right)$ as $x \rightarrow \infty$, uniformly in $y_{1} \leqq y \leqq y_{2}$.

Then, for each solution $w(z)$ of $(3.3),\|w(z)\|=O(h(x, y))$ as $x \rightarrow \infty$, 
uniformly in $y_{1} \leqq y \leqq y_{2}$, and there is a solution $W(z) c$ of (3.5) such that

$$
v(z)=W(z)(c+\varepsilon(z))
$$

in $R_{1}$, where $\|\varepsilon(z)\|$ tends to zero uniformly in $R_{1}$ as $z \rightarrow \infty$.

Proof. First we observe that $W(z) / h(z)$ is of $O(1)$ on the lines $y=y_{1}$, $y=y_{2}$, of $O\left(e^{e b x}\right)$ uniformly in $R_{1}$. According to Lemma $3, W(z) / h(z)$ is of $O(1)$ in $R_{1}$. It follows that

$$
\|W(z)\|=O(h(x, y))
$$

uniformly in $y_{1} \leqq y \leqq y_{2}$.

For a solution, $w(z)$, of $(3.3), w\left(z_{\jmath}\right)=c$, we have

$$
w(z)=W(z) c+\int_{z_{0}}^{z} W(z) W^{-1}(t)[B(t) w(t)+f(t)] d t,
$$

where $z$ and $z_{0}$ are in $R_{1}$. Multiplication of (3.9) by $W^{-1}(z)$ yields

$$
W^{-1}(z) w(z)=c+\int_{z_{0}}^{z} W^{-1}(t)[B(t) w(t)+f(t)] d t .
$$

Since

$$
\left\|\int_{x_{0}+i y}^{x+i y} W^{-1}(t) f(t) d t\right\| \leqq M K(x, y)
$$

for a suitable constant $M$, it follows that

$$
\int_{z_{\jmath}}^{z} W^{-1}(t) f(t) d t
$$

being analytic in $R_{1}$, is bounded in $R_{1}$, by virtue of the conditions in (c) and Lemma 3. Also the integral

$$
\int_{x_{0}+i y_{0}}^{x_{0}+i y} W^{-1}(t) B(t) w(t) d t
$$

is bounded in $R_{1}$. From (3.10), we then have

$$
W^{-1}(z) w(z)=E(z)+\int_{x_{0}+i y}^{x+i y} W^{-1}(t) B(t) w(t) d t
$$

for some function $E(z), E(z)$ being bounded in $R_{1}$, and the integral taken along a straight line. Write $w(t)=W(t) W^{-1}(t) w(t)$. Then

$$
\left\|W^{-1}(z) w(z)\right\| \leqq M+\int_{x_{0}+i y}^{x+i y}\left\|W^{-1}(t) B(t)\right\|\left\|W^{-1}(t) w(t)\right\| d t
$$

for some constant $M$, and $x \geqq x_{0}$. An appeal to Lemma 2 yields 


$$
\left\|W^{-1}(z) v(z)\right\| \leq M \exp \left[\int_{x_{0}+i \eta !}^{x+i, y}\left\|W^{-1}(t) B(t) W(t)\right\| d t\right], \quad x \geqslant x_{0} .
$$

In view of the definition of $H(x, y),(3.13)$ gives

$$
\left\|W^{-1}(z) w(z)\right\| \leq M e^{N \Pi(x, y)}
$$

for some constant $N$. It follows that $W^{-1}(z) w(z)$, being analytic in $R_{1}$, is bounded in $R_{1}$, by virtue of the conditions in (b) and Lemma 3 . Since

$$
\|w(z)\| \leqq\|W(z)\|\left\|W^{-1}(z) w(z)\right\|,
$$

we have also

$$
\|w(z)\|=O(h(x, y))
$$

as $x \rightarrow \infty$, uniformly in $y_{1} \leqq y \leqq y_{2}$. From (3.10), the analytic function

$$
\int_{z_{0}}^{z} W^{-1}(t)[B(t) w(t)+f(t)] d t
$$

is bounded in $R_{1}$. By virtue of (3.6), (3.7), (b) and (c), the integral (3.16) tends to a limit $c_{1}$ as $z \rightarrow \infty$ along $y=y_{1}$ and to a limit $c_{2}$ along $y=y_{2}$, and hence, by Lemma 4 , it tends to a limit $c_{0}$ uniformly in $R_{1}$ and $c_{0}=c_{1}=c_{2}$. Now (3.10) can be written as

$$
W^{-1}(z) w(z)=c-\int_{z}^{\infty} W^{-1}(t)[B(t) w(t)+f(t)] d t
$$

for some $c$, the integral being convergent to zero uniformly in $R_{1}$. is equivalent to (3.8). This proves the theorem.

THEOREM 4. Let the conditions of Theorem 3 be satisfied. Furthermore let

(a) $h(x, y)=O\left(e^{e^{b x}}\right)$ as $x \rightarrow \infty$, uniformly in $R_{1}$,

(b) $h\left(x, y_{i}\right) H\left(\infty, y_{i}, x\right)$ and $h\left(x, y_{i}\right) K\left(\infty, y_{i}, x\right)$ tend to zero $x \rightarrow \infty$, $i=1,2$.

Then, for each solution w(z) of (3.3), there is a solution $W(z)$ c of (3.5) such that

$$
w(z)=W(z) c+\varepsilon(z)
$$

in $R_{1}$, where $\|\varepsilon(z)\|$ tends to zero uniformly in $R_{1}$ as $z \rightarrow \infty$.

Proof. Using (a) and (b), clearly the analytic function

$$
\varepsilon(z)=W(z) \int_{z}^{\infty} W^{-1}(t)[B(t) w(t)+f(t)] d t
$$

converges to zero as $z \rightarrow \infty$ along the lines $y=y_{1}, y=y_{2}$ and

$$
\|\varepsilon(z)\|=O\left(e^{e^{b x}}\right) \text {, uniformly in } R_{1} .
$$


According to Lemma $3,\|\varepsilon(z)\|$ is bounded in $R_{1}$, and by Lemma 4 , $\|\varepsilon(z)\|$ converges to zero uniformly in $R_{1}$ as $\approx \rightarrow \infty$. From (3.17), we have

$$
w(z)=W(z) c-W(z) \int_{z}^{\infty} W^{-1}(t)[B(t) w(t)+f(t)] d t
$$

which is equivalent to (3.18). This completes the proof.

We shall state two similar theorems for the region $R_{2}$. Set

$$
\begin{aligned}
& L(r, \theta)=L\left(r, \theta, r_{0}\right)=\int_{r_{0}}^{r}[h(r, \theta)]^{n}\|B(z)\| \exp \left[-\mathscr{R} \int_{z_{0}}^{z} \operatorname{tr} A(u) d u\right] d r, \\
& J(r, \theta)=J\left(r, \theta, r_{0}\right)=\int_{r_{0}}^{r}[h(r, \theta)]^{n-1}\|f(z)\| \exp \left[-\mathscr{R} \int_{z_{0}}^{z} \operatorname{tr} A(u) d u\right] d r,
\end{aligned}
$$

where $z=r \exp (i \theta), z_{0}=r_{0} \exp \left(i \theta_{0}\right), z_{0}$ being in $R_{2}$.

THEOREM 5. Let

(a) $\|W(z)\|=O(h(r, \theta))$ as $r \rightarrow \infty$ for $\theta=\theta_{1}$ and $\theta=\theta_{2}$, $\|W(z)\|=O\left(h(r, \theta) e^{r^{b}}\right)$ as $r \rightarrow \infty$, uniformly in $\theta_{1} \leqq \theta \leqq \theta_{2}$, where $b<a, \theta_{2}-\theta_{1}=a^{-1} \pi, h(r, \theta)=|h(z)|$ and $h(z)$ is analytic in $R_{2}$,

(b) $L(r, \theta)=O(1)$ as $r \rightarrow \infty$ for $\theta=\theta_{1}$ and $\theta=\theta_{3}$, $L(r, \theta)=O\left(r^{b}\right)$ as $r \rightarrow \infty$, uniformly in $\theta_{1} \leqq \theta \leqq \theta_{2}$,

(c) $J(r, \theta)=O(1)$ as $r \rightarrow \infty$ for $\theta=\theta_{1}$ and $\theta=\theta_{2}$, $J(r, \theta)=O\left(e^{r^{b}}\right)$ as $r \rightarrow \infty$, uniformly in $\theta_{1} \leqq \theta \leqq \theta_{2}$.

Then, for each solution $w(z)$ of $(3.3),\|w(z)\|=O(h(r, \theta))$ as $r \rightarrow \infty$, uniformly in $\theta_{1} \leqq \theta \leqq \theta_{2}$, and there is a solution $W(z) c$ of (3.5) such that

$$
w(z)=W(z)(c+\varepsilon(z))
$$

in $R_{2}$, where $\|\varepsilon(z)\|$ tends to zero uniformly in $R_{2}$ as $z \rightarrow \infty$.

The proof is similar to that of Theorem 3.

TheOREm 6. Let the conditions of Theorem 5 be satisfied. Furthermore let

(a) $h(r, \theta)=O\left(e^{r^{b}}\right)$ as $r \rightarrow \infty$, uniformly in $R_{2}$,

(b) $h\left(r, \theta_{i}\right) L\left(\infty, \theta_{i}, r\right)$ and $h\left(r, \theta_{i}\right) J\left(\infty, \theta_{i}, r\right)$ tend to zero as $r \rightarrow \infty$, $i=1,2$.

Then for each solution $w(z)$ of (3.3) there is a solution $W(z) c$ of (3.5) such that

$$
w(z)=W(z) c+\varepsilon(z)
$$

in $R_{2}$, where $\|\varepsilon(z)\|$ tends to zero uniformly in $R_{2}$ as $z \rightarrow \infty$.

The proof of this theorem is similar to that of Theorem 4 . 
4. Second order differential equation. When the theorems in $\$ \S 2$ and 3 apply to a given second order differential equation, it is necessary to know the order relation of both the solutions and their derivatives of the approximate equation. Of course we obtain an asymptotic relation between the solutions, and their derivative of each equation. However, for second order differential equations, we are able to establish asymptotic relations just for the solutions when the order relation of the solution of the approximate equation is given. In view of the interest in second order differential equations, we state some similar results and briefly indicate the proofs.

Consider the second order differential equations

$$
\begin{aligned}
& \left(r(x) y^{\prime}\right)^{\prime}+p(x) y=f(x, y), \\
& \left(r(x) y^{\prime}\right)^{\prime}+p(x) y=0,
\end{aligned}
$$

where $1 / r(x), p(x)$ and $f(x, y)$ (for each fixed complex $y$ ) are complexvalued functions of the real variable $x$, defined for all $x \geqslant 0$ and belonging to $L(0, R)$ for every positive $R$; furthermore for each $x \geqq 0$ $f(x, y)$ is a continuous function of $y$ for all complex $y$ satisfying

$$
\left|f\left(x, y_{1}\right)-f\left(x, y_{2}\right)\right| \leqq g(x)\left|y_{1}-y_{2}\right|
$$

for all complex $y_{1}$ and $y_{2}, g(x)$ being of the class $L(0, R)$ for every positive $R$.

Let $y_{1}(x)$ and $y_{2}(x)$ be two linearly independent solutions of (4.2) with

$$
r(x)\left[y_{1}(x) y_{2}^{\prime}(x)-y_{1}^{\prime}(x) y_{2}(x)\right]=1
$$

almost everywhere.

Theorem 7. Let

(a) $\left|y_{1}(x)\right|+\left|y_{2}(x)\right|=O(h(x))$ as $x \rightarrow \infty, \quad h(x)$ being measurable on $0 \leqq x<\infty$,

(b) $[h(x)]^{2} g(x)$ and $h(x) f(x, 0)$ belong to $L(0, \infty)$.

Then, for each solution $y(x)$ of $(4.1), y(x)=O(h(x))$ as $x \rightarrow \infty$ and there exist constants $A$ and $B$ such that

$$
\begin{aligned}
& y(x)=(A+\varepsilon(x)) y_{1}(x)+\left(B+\varepsilon_{2}(x)\right) y_{2}(x), \\
& y^{\prime}(x)=\left(A+\varepsilon_{1}(x)\right) y_{1}^{\prime}(x)+\left(B+\varepsilon_{2}(x) y_{2}^{\prime}(x),\right.
\end{aligned}
$$

where $\varepsilon_{1}(x)$ and $\varepsilon_{2}(x)$ tend to zero as $x \rightarrow \infty$. (The second equation holds only almost everywhere.)

Proof. Let $y(x)$ be a solution of (4.1). Then, by the method of variation of parameters, $y(x)$ satisfies 


$$
\left.y(x)=A y_{1}(x)+B y_{2}(x)+\int_{11}^{x} f(t, y(t))\left[y_{2}(x)\right) y_{1}(t)-y_{1}(x) y y_{2}(t)\right] d t
$$

for some constants $A$ and $B$. Set $u=|y| /\left(\left|y_{1}\right|+\left|y_{2}\right|\right)$. Using (a), (b) and (4.3) one can verify that

$$
u(x) \leqq M+N \int_{0}^{x} g(t) h^{2}(t) u(t) d t
$$

for some positive constants $M$ and $N$ and $x \geq 0$. By Lemma 2 and (b), $u(x)$ is bounded on $0 \leq x<\infty$. It follows that $y(x)=O(h(x))$ as $x \rightarrow \infty$. Write

$$
\varepsilon_{1}(x)=\int_{x}^{\infty} f(t, y(t)) y_{1}(t) d t, \quad \varepsilon_{2}(x)=-\int_{x}^{\infty} f(t, y(t)) y_{1}(t) d t,
$$

the existence of the integrals being guaranteed by (b). The first part of (4.5) follows from (4.6) by splitting the integral, the second part from differentiation of the first.

THEOREM 8. Let

(a) $\left|y_{1}(x)\right|+\left|y_{2}(x)\right|=O(h(x))$ as $x \rightarrow \infty$, $h(x)$ being non-decreasing on $0 \leq x<\infty$,

(b) $h^{3}(x) g(x)$ and $h^{2}(x) f(x, 0)$ belong to $L(0, \infty)$.

Then for each solution $y(x)$ of (4.1) there exist constants $A$ and $B$ such that

$$
y(x)=A y_{1}(x)+B y_{0}(x)+\varepsilon(x),
$$

where $\varepsilon(x)$ tends to zero as $x \rightarrow \infty$.

Proof. Under conditions (a) and (b), $\varepsilon_{1}(x) y_{1}(x)$ and $\varepsilon_{2}(x) y_{2}(x)$ tend to zero as $x \rightarrow \infty$. (4.8) then follows from the first equation of (4.5).

Remark. In some cases, with properly chosen $y_{1}$ and $y_{2}$, condition (b) of Theorem 8 may be weakened. For instance let $r(x)=1, p(x)=0$ and take $y_{1}(x)=1, y_{2}(x)=x, h(x)=x$; it is true that $y(x)=A x+B+\varepsilon(x)$, $\varepsilon(x) \rightarrow 0$ as $x \rightarrow \infty$ if $h^{2}(x) g(x)$ and $h(x) f(x, 0)$ belong to $L(0, \infty)$. The discrepancy is due to the fact that in the general case we consider $y_{1}(x) y_{2}(x)=O\left(h^{2}(x)\right)$, while in this example $y_{1}(x) y_{2}(x)=O(h(x))$.

Consider the differential equations

$$
\left(r(z) w^{\prime}\right)^{\prime}+(p(z)+q(z)) w=f(z),
$$

$$
\left(r(z) v^{\prime}\right)^{\prime}+p(z) w=0,
$$

where $r(z) \neq 0, p(z), q(z)$ and $f(z)$ are single-valued analytic functions 
in $R_{1}$ or $R_{2}$ (see (3.1) and (3.2)), as whichever is concerned. Let $w_{1}(z)$ and $w_{2}(z)$ be two linearly inderendent solutions of (4.10) with

$$
r(z)\left[w_{1}(z) w_{2}^{\prime}(z)-w_{1}^{\prime}(z) w_{2}(z)\right]=1 .
$$

Set

$$
\begin{aligned}
& H(x, y)=H\left(x, y, x_{0}\right)=\int_{x_{j}}^{x} h^{2}(x, y)|q(z)| d x, \\
& K(x, y)=K\left(x, y, x_{0}\right)=\int_{x_{0}}^{x} h(x, y)|f(z)| d x,
\end{aligned}
$$

where $z=x+i y, z_{0}=x_{0}+i y_{0}, z_{0}$ being in $R_{1}$.

THEOREM 9. Let

(a) $\left|w_{1}(z)\right|+\left|w_{2}(z)\right|=O(h(x, y))$ as $x \rightarrow \infty$ for $y=y_{1}$ and $y=y_{2}$, $\left|w_{1}(z)\right|+\left|w_{2}(z)\right|=O\left(h(x, y) e^{e^{b x}}\right)$ as $x \rightarrow \infty$, uniformly in $y_{1} \leqq y \leqq y_{2}$, where $b<a, y_{2}-y_{1}=a^{-1} \pi, h(x, y)=|h(z)|$ and $h(z)$ is analytic in $R_{1}$,

(b) $H(x, y)=O(1)$ as $x \rightarrow \infty$ for $y=y_{1}$ and $y=y_{2}$, $H(x, y)=O\left(e^{n x}\right)$ as $x \rightarrow \infty$, uniformly in $R_{1}$,

(c) $K(x, y)=O(1)$ as $x \rightarrow \infty$ for $y=y_{1}$ and $y=y_{\text {, }}$, $K(x, y)=O\left(e^{e^{b x}}\right)$ as $x \rightarrow \infty$, uniformly in $R_{1}$.

Then, for each solution $w(z)$ of $(4.9), w(z)=O(h(x, y))$ as $x \rightarrow \infty$, uniformly in $y_{1} \leq y \leq y_{2}$, and there exist constants $A$ and $B$ such that

$$
\begin{aligned}
& w(z)=\left(A+\varepsilon_{1}(z)\right) w_{1}(z)+\left(B+\varepsilon_{2}(z)\right) w_{.}(z), \\
& w^{\prime}(z)=\left(A+\varepsilon_{1}(z)\right) w_{1}^{\prime}(z)+\left(B+\varepsilon_{2}(z)\right) w_{2}^{\prime}(z),
\end{aligned}
$$

in $R_{1}$, where $\varepsilon_{1}(z)$ and $\varepsilon_{2}(z)$ tend to zero uniformly in $R_{1}$ as $z \rightarrow \infty$.

THEOREM 10. Let the conditions of Theorem 9 be satisfied. Furthermore let

(a) $h(x, y)=O\left(e^{e^{b . x}}\right)$ as $x \rightarrow \infty$, uniformly in $R_{1}$,

(b) $h\left(x, y_{i}\right) H\left(\infty, y_{i}, x\right)$ and $h\left(x, y_{i}\right) K\left(\infty, y_{i}, x\right)$ tend to zero as $x \rightarrow \infty$, $i=1,2$.

Then for each solution $w(z)$ of (4.9) there exist constants $A$ and $B$ such that

$$
w(z)=A w_{1}(z)+B w_{2}(z)+\varepsilon(z)
$$

in $R_{1}$, where $\varepsilon(z)$ tends to zero uniformly in $R_{1}$ as $z \rightarrow \infty$.

Set

$$
L(r, \theta)=L\left(r, \theta, r_{0}\right)=\int_{r_{0}}^{r} h^{2}(r, \theta)|q(z)| d r,
$$




$$
J(r, \theta)=J\left(r, \theta, r_{0}\right)=\int_{r_{0}}^{r} h(r, \theta)|f(z)| d r .
$$

where $z=r \cdot \exp (i \theta), z_{0}=r_{0} \exp \left(i \theta_{0}\right), z_{0}$ being in $R_{2}$.

THEOREM 11. Let

(a) $\left|w_{1}(z)\right|+\left|w_{2}(z)\right|=O(h(r, \theta))$ as $r \rightarrow \infty$ for $\theta=\theta_{1}$ and $\theta=\theta_{2}$, $\left|w_{1}(z)\right|+\left|w_{2}(z)\right|=O\left(h(r, \theta) e^{r^{b}}\right)$ as $r \rightarrow \infty$, uniformly in $\theta_{1} \leqq \theta \leqq \theta_{2}$, where $b<a, \theta_{2}-\theta_{1}=a^{-1} \pi, h(r, \theta)=|h(z)|$ and $h(z)$ is analytic in $R_{2}$,

(b) $L(r, \theta)=O(1)$ as $r \rightarrow \infty$ for $\theta=\theta_{1}$ and $\theta=\theta_{2}$, $L(r, \theta)=O\left(r^{b}\right)$ as $r \rightarrow \infty$, uniformly in $R_{2}$,

(c) $J(r, \theta)=O(1)$ as $r \rightarrow \infty$ for $\theta=\theta_{1}$ and $\theta=\theta_{2}$, $J(r, \theta)=O\left(e^{r^{b}}\right)$ as $r \rightarrow \infty$, uniformly in $R_{2}$.

Then for each solution $w(z)$ of (4.9) there exist constants $A$ and $B$ such that

$$
\begin{aligned}
& w(z)=\left(A+\varepsilon_{1}(z)\right) w_{1}(z)+\left(B+\varepsilon_{2}(z)\right) w_{2}(z), \\
& w^{\prime}(z)=\left(A+\varepsilon_{1}(z)\right) w_{1}^{\prime}(z)+\left(B+\varepsilon_{2}(z)\right) w_{2}^{\prime}(z),
\end{aligned}
$$

in $R_{2}$, where $\varepsilon_{1}(z)$ and $\varepsilon_{2}(z)$ tend to zero uniformly in $R_{2}$ as $z \rightarrow \infty$.

TheOREm 12. Let the conditions of Theorem 11 be satisfied. Furthermore let

(a) $h(r, \theta)=O\left(e^{r^{b}}\right)$ as $r \rightarrow \infty$, uniformly in $R_{2}$,

(b) $h\left(r, \theta_{i}\right) L\left(\infty, \theta_{i}, r\right)$ and $h\left(r, \theta_{i}\right) J\left(\infty, \theta_{i}, r\right)$ tend to zero as $r \rightarrow \infty$, $i=1,2$.

Then for each solution $w(z)$ of (4.9) there exist constants $A$ and $B$ such that

$$
w(z)=A w_{1}(z)+B v_{2}(z)+\varepsilon(z)
$$

in $R_{y}$, where $\varepsilon(z)$ tends to zero uniformly in $R_{z}$ as $z \rightarrow \infty$.

Combining the methods of $\S 3$ and the first part of this section, one can prove these theorems by using the integral equation

$$
w(z)=A w_{1}(z)+B w_{2}(z)+\int_{z_{0}}^{z}[f(t)-q(t) w(t)]\left[w_{2}(z) w_{1}(t)-w_{1}(z) w_{2}(t)\right] d t
$$

which is satisfied by the solutions of (4.9). The details will be omitted.

\section{REFERENCES}

1. A. Wintner, Asymptotic equilibria, Amer. J. Math., 68 (1946), 125-132.

2. N. Levinson, The asymptotic behavior of a s!ste'm of limear differential equations, Amer. J. Math., 68 (1946), 1-6.

3. H. Weyl, Comment on the preceding paper, Amer. J. Math., 68 (1946), 7-12. 
4. E. C. Titchmarsh, The theory of functions, Oxford, 1944.

5. E. J. McShane, Integration, Princeton, 1947.

6. G. D. Birkhoff and R. E. Langer, The boundary problems and developmonts associatod with a system of ordinary differcntial cquations of first order, Proc. Amer. Acad. Arts and Sciences, 58 (1923), 49-128.

The Catholic University of America 


\section{PACIFIC JOURNAL OF MATHEMATICS}

EDITORS

\author{
H. L. Royden \\ Stanford University \\ Stanford, California \\ E. Hewite \\ University of Washington \\ Seattle 5 , Washington
}

\author{
R. P. Dilworth \\ California Institute of Technology \\ Pasadena 4, California
}

\author{
A. Horn* \\ University of California \\ Los Angeles 24, California
}

\section{ASSOCIATE EDITORS}
E. F. BECKENBACH
M. HALL
M. S. KNEBELMAN
J. J. STOKER
C. E. BURGESS
P. R. HALMOS
I. NIVEN
V. GANAPATHY IYER
T. G. OSTROM
G. SZEKERES
H. BUSEMANN
R. D. JAMES
M. M. SCHIFFER
F. WOLF
H. FEDERER

\section{SPONSORS}

\author{
UNIVERSITY OF BRITISH COLUMBIA \\ CALIFORNIA INSTITUTE OF TECHNOLOGY \\ UNIVERSITY OF CALIFORNIA \\ CALIFORNIA RESEARCH CORPORATION \\ MONTANA STATE UNIVERSITY \\ UNIVERSITY OF NEVADA \\ OREGON STATE COLLEGE \\ UNIVERSITY OF OREGON \\ UNIVERSITY OF SOUTHERN CALIFORNIA
}

\author{
STANFORD UNIVERSITY \\ UNIVERSITY OF UTAH \\ WASHINGTON STATE COLLEGE \\ UNIVERSITY OF WASHINGTON \\ AMERICAN MATHEMATICAL SOCIETY \\ HUGHES AIRCRAFT COMPANY
}

Mathematical papers intended for publication in the Pacific Journal of Mathematics should be typewritten (double spaced), and the author should keep a complete copy. Manuscripts may be sent to any of the editors. Manuscripts intended for the outgoing editors should be sent to their successors. All other communications to the editors should be addressed to the managing editor, Alfred Horn at the University of California, Los Angeles 24, California.

50 reprints of each article are furnished free of charge; additional copies may be obtained at cost in multiples of 50 .

The Pacific Journal of Mathematics is published quarterly, in March, June, September, and December. The price per volume (4 numbers) is $\$ 12.00$; single issues, $\$ 3.50$. Back numbers are available. Special price to individual faculty members of supporting institutions and to individual members of the American Mathematical Society: $\$ 4.00$ per volume; single issues, $\$ 1.25$.

Subscriptions, orders for back numbers, and changes of address should be sent to Pacific Journal of Mathematics, c/o University of California Press, Berkeley 4, California.

Printed at Kokusai Bunken Insatsusha (International Academic Printing Co., Ltd.), No. 10, 1-chome, Fujimi-cho, Chiyoda-ku, Tokyo, Japan.

* During the absence of E. G. Straus.

PUBLISHED BY PACIFIC JOURNAL OF MATHEMATICS, A NON-PROFIT CORPORATION COPYRIGHT 1956 BY PACIFIC JOURNAL OF MATHEMATICS 


\section{Pacific Journal of Mathematics}

\section{Vol. 6, No. $2 \quad$ December, 1956}

Louis Auslander, Remark on the use of forms in variational calculations .......................................... 209

Hubert Spence Butts, Jr. and Henry B. Mann, Corresponding residue systems in algebraic number fields ........................ 211

L. Carlitz and John Herbert Hodges, Distribution of matrices in a finite field............................................

Paul Civin and Bertram Yood, Invariant functionals ............... 231

David James Dickinson, Henry Pollak and G. H. Wannier, On a class of polynomials orthogonal over a denumerable set .................

Bernard Friedman and Luna Mishoe, Eigenfunction expansions associated with a non-self-adjoint differential equation ....................

Luna Mishoe and G. C. Ford, On the uniform convergence of a certain eigenfunction series .............................. 271

John W. Green, Mean values of harmonic functions on homothetic curves...........................................

Charles John August Halberg, Jr. and Angus E. Taylor, On the spectra of linked operators .....................................

Chuan Chih Hsiung, Some integral formulas for closed hypersurfaces in Riemannian space ................................... 291

Norman D. Lane, Differentiable points of arcs in conformal n-space 301

Louis F. McAuley, A relation between perfect separability, completeness, and normality in semi-metric spaces

G. Power and D. L. Scott-Hutton, The slow shearing motion of a liquid past a semi-infinite plane .............................

A. C. Schaeffer, Entire functions

Edward Silverman, An intrinsic inequality for Lebesgue area...

Choy-Tak Taam, Asymptotic relations between systems of differential equations.

Ti Yen, Quotient algebra of a finite $A W^{*}$-algebra ... 